ARTICLE

\title{
Scalable continuous evolution for the generation of diverse enzyme variants encompassing promiscuous activities
}

Gordon Rix ${ }^{1}$, Ella J. Watkins-Dulaney ${ }^{2}$, Patrick J. Almhjell ${ }^{3}$, Christina E. Boville ${ }^{2,6}$, Frances H. Arnold (D) ${ }^{2,3} \&$ Chang C. Liu (1D $1,4,5 \bowtie$

Enzyme orthologs sharing identical primary functions can have different promiscuous activities. While it is possible to mine this natural diversity to obtain useful biocatalysts, generating comparably rich ortholog diversity is difficult, as it is the product of deep evolutionary processes occurring in a multitude of separate species and populations. Here, we take a first step in recapitulating the depth and scale of natural ortholog evolution on laboratory timescales. Using a continuous directed evolution platform called OrthoRep, we rapidly evolve the Thermotoga maritima tryptophan synthase $\beta$-subunit (TmTrpB) through multimutation pathways in many independent replicates, selecting only on TmTrpB's primary activity of synthesizing L-tryptophan from indole and L-serine. We find that the resulting sequence-diverse $\operatorname{Tm} \operatorname{TrpB}$ variants span a range of substrate profiles useful in industrial biocatalysis and suggest that the depth and scale of evolution that OrthoRep affords will be generally valuable in enzyme engineering and the evolution of biomolecular functions.

\footnotetext{
${ }^{1}$ Department of Molecular Biology and Biochemistry, University of California, Irvine, CA, USA. ${ }^{2}$ Division of Biology and Biological Engineering, California Institute of Technology, Pasadena, CA, USA. ${ }^{3}$ Division of Chemistry and Chemical Engineering, California Institute of Technology, Pasadena, CA, USA. ${ }^{4}$ Department of Biomedical Engineering, University of California, Irvine, CA, USA. ${ }^{5}$ Department of Chemistry, University of California, Irvine, CA, USA.

${ }^{6}$ Present address: Aralez Bio, Emeryville, CA, USA. ${ }^{凶}$ email: ccl@uci.edu
} 
$\mathrm{N}$ atural enzymes typically have many orthologs. While the primary activity of orthologous enzymes is largely the same ${ }^{1}$, promiscuous functions not under selective pressure can vary widely ${ }^{2,3}$. Such variation may be attributed to the deep and distinct evolutionary histories shaping each ortholog, including long periods of neutral drift, recalibration of primary activity, and adaptation to new host environments such as temperature. These rich histories act to produce extensive genetic diversity, which underpins different promiscuity profiles ${ }^{2}$.

Diversity in promiscuous functions across orthologs is of both fundamental and practical importance. An enzyme's reserve of promiscuous activities dictates what secondary reactions, environmental changes, or niches the enzyme can accommodate ${ }^{4,5}$. Diversity in promiscuous activities therefore contributes to the basic robustness of life and adaptation. An enzyme's reserve of promiscuous activities can also be mined in the application of enzymes for biocatalysis ${ }^{6,7}$. Ortholog diversity therefore expands the range of reactions at the disposal of enzyme engineers, supporting the growing role of "green" enzymatic processes in the chemical and pharmaceutical industries ${ }^{8-10}$.

Inspired by the remarkable ability of enzyme orthologs to encompass promiscuous activities, we asked whether we could extend the substrate scope of useful enzymes by evolving multiple versions of an enzyme in the laboratory, selecting only for its primary function. Although this idea has been explored before using classical directed evolution approaches, most notably through the generation of cryptic genetic variation with neutral drift libraries ${ }^{11-14}$, we recognized that our recently developed continuous evolution system, OrthoRep, may be considerably better poised for this challenge ${ }^{15,16}$. Classical directed evolution mimics evolution through an iterative procedure that involves diversifying a gene of interest (GOI) in vitro (e.g., through errorprone PCR), transforming the resulting GOI library into cells, and selecting or screening for desired activities, where each cycle of this procedure represents one step in an evolutionary search ${ }^{17}$. However, since each cycle is manually staged, classical directed evolution does not readily admit depth and scale during exploration of functional sequence space-it is difficult to carry out many iterations to mimic lengthy evolutionary searches (depth), let alone do so in many independent experiments (scale). Yet evolutionary depth and scale are precisely the two characteristics responsible for ortholog diversity in nature. Natural orthologs have diversified from their ancestral parent over great evolutionary timescales, allowing for the traversal of long mutational pathways shaped by complex selection histories (depth). Natural orthologs are also the result of numerous independent evolutionary lineages, since spatially separated species and populations are free to take divergent mutational paths and experience different environments (scale). Systems that better mimic the depth and scale of natural enzyme evolution, but on laboratory timescales, are thus needed for the effective generation of enzyme variants that begin to approach the genetic and promiscuity profile diversity of orthologs.

OrthoRep is such a system. In OrthoRep, an orthogonal errorprone DNA polymerase durably hypermutates an orthogonal plasmid (p1) without raising the mutation rate of the host Saccharomyces cerevisiae genome ${ }^{16}$. Thus, GOIs encoded on $\mathrm{p} 1$ rapidly evolve when cells are simply passaged under selection. By reducing the manual stages of classical directed evolution down to a continuous process where cycles of diversification and selection occur autonomously in vivo, OrthoRep readily accesses depth and scale in evolutionary search ${ }^{16,18}$. Here, we apply OrthoRep to the evolution of the Thermotoga maritima tryptophan synthase $\beta$ subunit $(\operatorname{Tm} \operatorname{TrpB})$ in multiple independent continuous evolution experiments, each carried out for at least 100 generations. While we only pressure $\operatorname{Tm} \operatorname{TrpB}$ to improve its primary activity of coupling indole and serine to produce tryptophan, the large number of independent evolution experiments we ran (scale) and the high degree of adaptation in each experiment (depth) resulted in a panel of variants encompassing expanded promiscuous activity with indole analogs. In addition to the immediate value of these evolved $T m \operatorname{TrpBs}$ in the synthesis of tryptophan analogs, our study offers a template for enzyme engineering where evolutionary depth and scale is leveraged on laboratory timescales to generate effective variant collections covering broad substrate scope.

\section{Results}

Establishing a selection system for the evolution of TmTrpB variants. To evolve $\operatorname{Tm} \operatorname{TrpB}$ variants using OrthoRep, we first needed to develop a selection where yeast would rely on TmTrpB's primary enzymatic activity for growth. TmTrpB catalyzes the PLP-dependent coupling of L-serine and indole to generate L-tryptophan ( $\operatorname{Trp})$ in the presence of the tryptophan synthase a-subunit, $\operatorname{Tm} \operatorname{TrpA}{ }^{19}$. In T. maritima and all other organisms that contain a heterodimeric tryptophan synthase complex, TrpA produces the indole substrate that $\operatorname{TrpB}$ uses and the absence of $\operatorname{Trp} A$ significantly attenuates the activity of $\operatorname{TrpB}$ through loss of allosteric activation ${ }^{19,20}$. TRP5 is the $S$. cerevisiae homolog of this heterodimeric enzyme complex, carrying out both TrpA and TrpB reactions and producing Trp for the cell. We reasoned that by deleting the TRP5 gene and forcing $S$. cerevisiae to rely on $\operatorname{Tm} \operatorname{TrpB}$ instead, cells would be pressured to evolve high stand-alone $T m \operatorname{TrpB}$ activity in order to produce the essential amino acid Trp in indole-supplemented media (Fig. 1a). This selection pressure would also include thermoadaptation, as yeast grow at mesophilic temperatures in contrast to the thermophilic source of $\operatorname{Tm} \operatorname{TrpB}$. Therefore, the selection on TmTrpB's primary activity would be multidimensional-standalone function, temperature, and neutral drift implemented when desired - and could result in complex evolutionary pathways that serve our goal of maximizing functional variant diversity across replicate evolution experiments. In addition, the multidimensional selection also serves practical goals as stand-alone activity is useful in biosynthetic applications (enzyme complexes are difficult to express and use in vitro) and activity at mesophilic temperatures is more compatible with heat-labile substrates, industrial processes where heating costs can compound, or in vivo applications in model mesophilic hosts (e.g. S. cerevisiae or Escherichia coli).

To test this selection, we turned to a positive control $\operatorname{Tm} \operatorname{TrpB}$ variant called TmTriple. This variant was previously engineered to enable stand-alone activity, free from dependence on allosteric activation by $\operatorname{TrpA}$, through a minimal set of three mutations ${ }^{7}$. We found that TmTriple rescued TRP5 function in a $\Delta \operatorname{trp} 5$ strain in an indole-dependent manner, validating our selection (Supplementary Fig. 1). Notably, TmTriple, along with other TrpB variants tested, only supported complementation when expressed from a high-strength promoter (Supplementary Fig. 1). This highlighted the opportunity for substantial adaptation and drift even in evolution experiments that start from already engineered $\operatorname{Tm} \operatorname{TrpB}$ variants.

Continuous evolution of $\operatorname{Tm} \operatorname{TrpB}$ with depth and scale. We encoded wild-type (wt) $\operatorname{Tm} \operatorname{TrpB}$, TmTriple, as well as a nonsense mutant of TmTriple, TmTripleQ90*, onto OrthoRep's p1 plasmid, which is replicated by a highly error-prone orthogonal DNA polymerase. TmTripleQ90* was included because reversion of the stop codon at position 90 in TmTripleQ90* would act as an early indication that adaptation was occurring, giving us confidence to continue passaging our evolution experiments for several weeks 
a
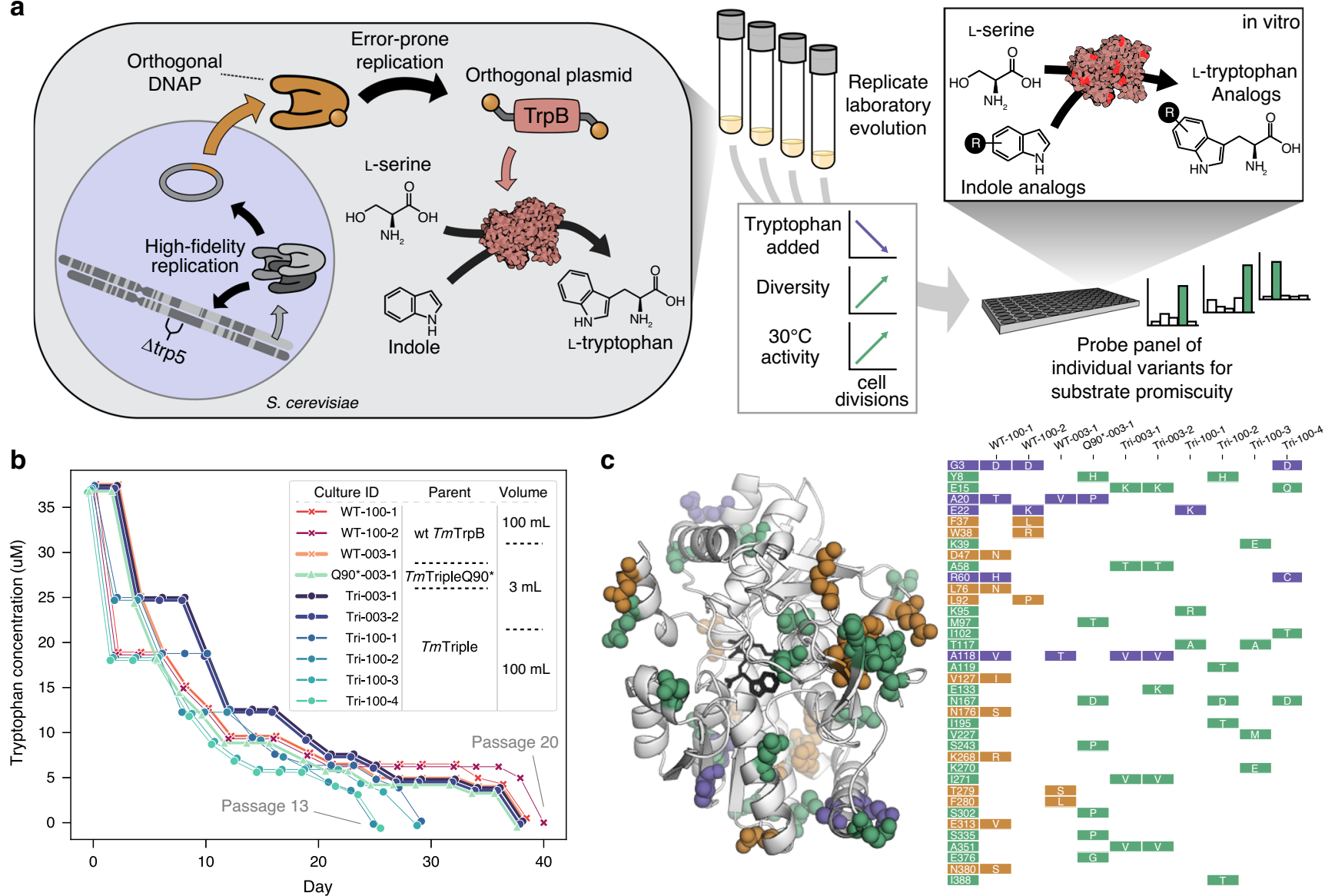

Fig. 1 OrthoRep-mediated continuous in vivo evolution of $\operatorname{Tm} T r p B$ to generate many diverse, functional variants. a Pipeline for the use of OrthoRep continuous directed evolution to generate many diverse, functional TmTrpB sequences. TmTrpB variants are first evolved in replicate for Trp production in yeast. OrthoRep enables replicate evolution through error-prone replication of an orthogonal plasmid by an orthogonal polymerase, maintaining low error rates in genome replication. By encoding a TmTrpB variant on this plasmid in a tryptophan synthase (TRP5) deletion mutant, TmTrpB may be both continuously diversified and selected for through gradual reduction in Trp supplied in the growth medium. Evolved populations containing many diverse, functional individuals may then be randomly sampled and tested for activity with indole analogs. $\operatorname{Tm} \operatorname{TrpB}$ illustration generated using 1 llustrate ${ }^{40}$.

b Selection trajectories for ten replicate cultures that evolved sufficient $\operatorname{Tm} \operatorname{TrpB}$ activity to support cell growth without supplemented Trp. Each point represents a single 1:100 dilution (passage) into fresh indole-supplemented growth medium. Trp concentration of fresh media was reduced when high saturation was achieved in the previous passage. Plots are slightly offset from true values to allow for visibility of all selection trajectories. Tm3C and Tm3D are plotted as one line as their trajectories were identical. c TmTrpB homology model and table depicting consensus mutations of the ten cultures shown in b. Mutations are colored by their appearance in populations evolved from wt TmTrpB (orange), TmTriple or TmTripleQ90* (green), or both (purple).

to maximize evolutionary search depth. In all three OrthoRep $\Delta \operatorname{trp} 5$ strains, the initial $\operatorname{Tm} \operatorname{TrpB}$ sequences enabled only minimal indole-dependent complementation (Supplementary Fig. 1). This was expected for wt $\operatorname{Tm} \operatorname{TrpB}$, which has low stand-alone enzymatic activity and TmTripleQ90*, which has a premature stop codon; and was unsurprising for TmTriple, since TmTriple displayed indole-dependent complementation only when artificially overexpressed (Supplementary Fig. 1).

To continuously evolve TmTrpB, we passaged cells encoding wt TmTrpB, TmTriple, or TmTripleQ90* on OrthoRep in the presence of $100 \mu \mathrm{M}$ indole while reducing the amount of Trp in the medium over time. In total, six $100 \mathrm{~mL}$ and twenty $3 \mathrm{~mL}$ cultures were passaged, each representing a single independent evolutionary trajectory. Passages were carried out as 1:100 dilutions where Trp concentrations were decreased in the $(\mathrm{N}+$ 1)th passage if cells grew quickly in the $\mathrm{N}^{\text {th }}$ passage, until Trp was fully omitted. All six of the $100 \mathrm{~mL}$ cultures, and four of the twenty $3 \mathrm{~mL}$ cultures fully adapted and were capable of robust growth in indole-supplemented media lacking Trp after 90-130 generations (13-20 passages) (Fig. 1b, Supplementary Table 1).
Populations that did not achieve growth in the absence of Trp still adapted, but stopped improving at $\sim 5 \mu \mathrm{M}$ supplemented Trp, suggesting a suboptimal local fitness maximum that is more easy to escape through the greater sequence diversity represented in larger populations. This could explain the different success rates in reaching full adaptation between the $3 \mathrm{~mL}$ and $100 \mathrm{~mL}$ populations. Cultures that did adapt fully were passaged for an additional $\sim 40$ generations without increasing selection stringency to allow for accumulation of further diversity through neutral drift.

For each of the 10 fully adapted populations, we PCR-amplified and bulk-sequenced the $\operatorname{Tm} \operatorname{TrpB}$ alleles on the p1 plasmid. Mutations relative to the parent $\operatorname{Tm} \operatorname{TrpB}$ variant detected at $>50 \%$ frequency in each population were deemed consensus mutations for that population, with the exception of reversion of the stop codon in populations evolving TmTripleQ90*. This stop codon reversion occurred at $100 \%$ frequency in the relevant populations and was not counted in any subsequent analyses due to its triviality. An average of $5.6( \pm 2.3$ s.d.) and a range of $3-11$ consensus amino acid changes per population were observed 


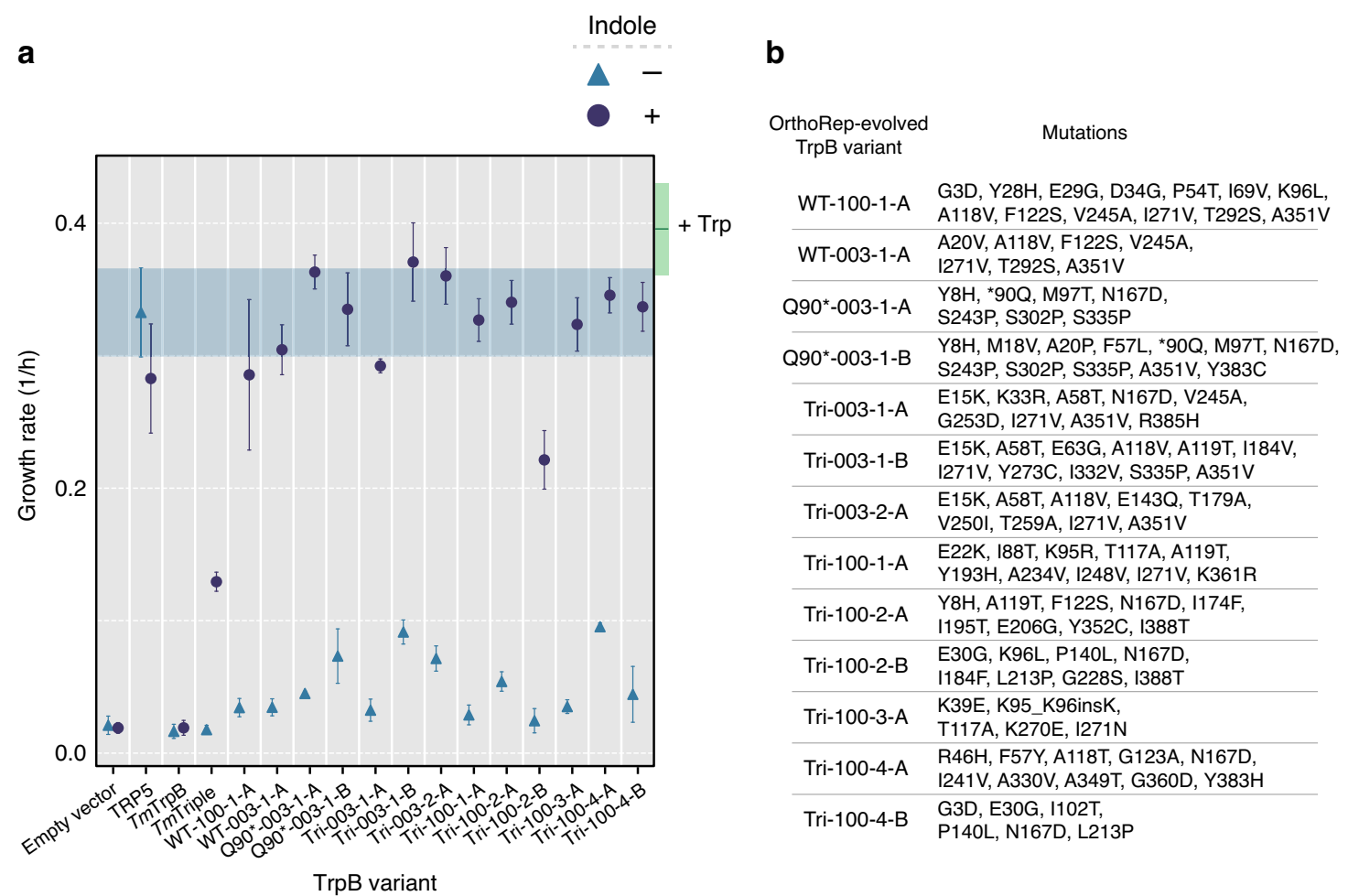

Fig. 2 In vivo activity and diversity of individual TmTrpB variants from OrthoRep-evolved populations. a Evaluation of TRP5 complementation by evolved variants through a growth rate assay. Maximum growth rates over a 24-h period for $\Delta$ trp5 yeast strains transformed with a nuclear plasmid expressing the indicated $\operatorname{Tm} \operatorname{TrpB}$ variant, grown in medium with or without $400 \mu \mathrm{M}$ indole. Points and error bars represent mean \pm s.d. for four biological replicates, respectively. Shaded area is the mean \pm s.d. growth rate for the TRP5 positive control (i.e. plasmid expressing the endogenous yeast TRP5). Green box indicates the mean \pm s.d. growth rate for all strains shown when Trp is supplemented. Growth rates for individual replicates in all three media conditions are shown in Supplementary Fig. 2. Note that growth rates below 0.15 per hour correspond to cultures that did not enter exponential phase; in these cases, the reported growth rate is not meaningful and instead can be interpreted as no quantifiable growth. b Parent populations from which OrthoRep-evolved variants shown in a are derived and all non-synonymous mutations present in each.

(Fig. 1c and Supplementary Table 2). Some of these mutations occurred at residues previously identified as relevant in

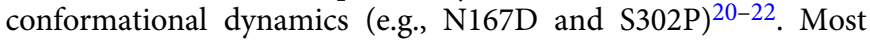
mutations observed, however, have not been previously identified in laboratory engineering experiments, suggesting that even the consensus of these populations explored uncharacterised regions of $\operatorname{Tm} T r \mathrm{r} B$ 's fitness landscape, doing so with diversity across replicates (Fig. 1c) that might translate to diversity in promiscuous activities across evolved variants.

Evolved TmTrpB variants improve Trp production in vivo and contain cryptic genetic variation. To ensure that evolved $T m \operatorname{TrpB}$ variants, and not potential host genomic mutations, were primarily responsible for each population's adaptation, we cloned individual TmTrpBs into a standard low copy yeast nuclear plasmid under a promoter that approximates expression from $\mathrm{p}^{23,24}$, transformed the variants into a fresh $\Delta \operatorname{trp} 5$ strain, and tested for their ability to support indole-dependent growth in the absence of Trp (Fig. 2 and Supplementary Fig. 2). Sixteen $T m \operatorname{TrpB}$ mutants were tested, representing one or two individual variants from each of the ten fully adapted populations. We found that 12 of the $16 \operatorname{TrpB}$ variants complemented growth to a similar degree as TRP5 when supplemented with $400 \mu \mathrm{M}$ indole, demonstrating substantial improvement over their wt $\operatorname{Tm} \operatorname{TrpB}$ and TmTriple parents (Fig. 2a).

Unsurprisingly, this set of clonal $\operatorname{Tr} \operatorname{TrpBs}$ contained more sequence diversity than the consensus sequences of the ten populations from which they were taken. Together, the variants tested comprised a total of 85 unique amino acid substitutions, with an average of $8.7( \pm 2.1$ s.d.) and a range of 5-13 nonsynonymous mutations per variant (variant set 1 , Supplementary Tables 2 and 3). Since the $12 \operatorname{Tm}$ TrpBs from this set exhibiting complementation were all similarly active in their primary activity yet mutationally diverse (Fig. 2b), we may conclude that our scaled evolution experiments generated substantial cryptic genetic variation. We note that four of $16 T m T r p B$ variants exhibited similar or lower Trp productivity compared to their parent (Supplementary Fig. 2). We suspect that the multicopy nature of $\mathrm{p} 1$ in the OrthoRep system allowed for deleterious mutations that appeared toward the end of the experiment to be maintained for a period of time without experiencing purifying selection if they arose in the same cell as functional variants, explaining the presence of these low activity $\operatorname{Tm} \operatorname{TrpBs}$. Indeed, this multicopy "buffering" may have worked to our advantage by promoting genetic drift under selection, facilitating both greater adaptation and greater diversity of evolutionary pathways across replicates (see "Discussion"). This may partly account for the high activity and high cryptic genetic variation present in the evolved TmTrpBs.

Evolved TmTrpBs exhibit high primary and promiscuous activity in vitro. We further characterized the evolved TmTrpBs in vitro to approximate conditions of industrial application, make kinetic measurements, and test whether promiscuous activity could be detected. Nine $\operatorname{Tm} \operatorname{TrpB}$ variants were sampled from those that supported robust indole-dependent growth in the $\Delta \operatorname{trp} 5$ strain, cloned into an E. coli expression vector with a Cterminal polyhistidine tag, and overexpressed. To mimic 
streamlined purification conditions compatible with biocatalytic application of $\operatorname{Tm} \operatorname{TrpBs}$, we generated heat treated E. coli lysates $\left(1 \mathrm{~h}\right.$ incubation at $\left.75^{\circ} \mathrm{C}\right)$ and tested them for their ability to couple indole and serine to produce $\operatorname{Trp}$ at $30^{\circ} \mathrm{C}$. Three of the nine OrthoRep-evolved TrpBs, WT-003-1-A, Q90*-003-1-A, and Tri-100-2-A, demonstrated improved activity over the benchmark TmTriple, as measured by total turnover number (TTN) (Supplementary Fig. 3). Conveniently, each of these variants was evolved from a different starting point, meaning that wt $\operatorname{Tm} \operatorname{TrpB}$, TmTriple, and TmTripleQ90* were all viable starting points for reaching high activity TmTrpBs. (See Supplementary Table 3 for an explanation of variant naming conventions where each name designates the source of the variant. For example, WT-003-1-A designates variant $A$ taken from the first replicate of a $3 \mathrm{~mL}$ evolution experiment starting from wt $\operatorname{Tm} \operatorname{TrpB}$.).

Since the benchmark TmTriple against which we compared the evolved TmTrpBs was engineered through classical directed evolution involving screening E. coli lysates, whereas our TmTrpB variants were evolved in yeast but expressed in E. coli for characterization, it is likely that the high-activity evolved TmTrpBs would compare even more favorably if normalized by expression. We therefore purified WT-003-1-A, Q90*-003-1-A, and Tri-100-2-A by immobilized metal affinity chromatography (IMAC) and reevaluated their activity for coupling indole with serine to generate Trp. By TTN, all three variants showed a 4- to 5 -fold increase in activity over $\mathrm{Tm}$ Triple at $30^{\circ} \mathrm{C}$ (Supplementary Fig. 4). At $75^{\circ} \mathrm{C}$, however, WT-003-1-A had only $\sim 2$-fold higher activity than TmTriple, while the other two variants were less active than TmTriple. Since the thermostability of WT-003-1-A, Q90*-003-1-A, and Tri-100-2-A had not been reduced dramatically $\left(T_{50}>83.7^{\circ} \mathrm{C}\right.$, Supplementary Fig. 5), adaptation in these variants occurred at least partially by shifting the activity temperature profile. This is a practically valuable adaptation since thermostable enzymes that operate at mesophilic temperatures allow for greater versatility in application without sacrificing durability and ease of purification through heat treatment.

Further testing of WT-003-1-A, Q90*-003-1-A, and Tri-100-2A revealed that all three enzymes had at least a 22 -fold higher $k_{\text {cat }} / K_{\mathrm{M}}$ for indole than did Tm Triple at $30^{\circ} \mathrm{C}$ (Supplementary Table 4 and Supplementary Fig. 6). Finally, testing for production of Trp analogs revealed that these variants' improved performance with indole transferred to alternate substrates (Supplementary Fig. 4), validating their utility as versatile biocatalysts and also the hypothesis that continuous evolution of $\operatorname{Tm} \operatorname{TrpB}$ variants can uncover promiscuous activities for which they were not selected.

A diverse panel of evolved $T m$ TrpB variants encompasses a variety of useful promiscuous activities with indole analogs. Given the exceptional performance of WT-003-1-A, Q90*-003-1A, and Tri-100-2-A and their ability to transfer primary activity to new substrates as promiscuous activity, we decided to further sample the variant diversity generated across the multiple TmTrpB evolution experiments. We cloned 60 randomly chosen $T m$ TrpBs from the ten continuous evolution populations into $E$. coli expression vectors for in vitro characterization. These 60 $T m$ TrpBs represent extensive diversity, with an average of 9.3 $( \pm 2.8$ s.d.) non-synonymous mutations per variant and a total of 194 unique amino acid changes across the set; in addition, each sequence encoded a unique protein (variant set 2, Supplementary Tables 2 and 3). Since each variant had multiple nonsynonymous mutations (up to 16) accumulated through $>100$ generations of adaptation and neutral drift, the depth of OrthoRep-based evolution was indeed leveraged in their evolution. We visualized these sequences, together with the consensus sequences of the populations from which they were derived, as nodes in a force directed graph related by shared mutations (Supplementary Fig. 7). With only one exception, all individual sequences cluster near the consensus sequence for their population, meaning that interpopulation diversity exceeded intrapopulation diversity. Thus, the scale of OrthoRep-based evolution was also leveraged in these variants - if fewer independent evolution experiments had been run, the reduction in diversity would not be recoverable from sampling more clones.

Preparations of TmTrpBs WT-003-1-A, Q90*-003-1-A, and Tri-100-2-A, the 60 new variants, and four top-performing $\operatorname{TrpB}$ benchmark variants from past classical directed evolution campaigns (including TmTriple) were all tested for product formation with indole by UV absorption and nine indole analogs by high performance liquid chromatography-mass spectrometry (HPLC-MS) to detect substrate promiscuity (Fig. 3a). The panel of 63 OrthoRep-evolved TmTrpB variants exhibited an impressive range of activities (Fig. 3b). First, we observed that a number of variants had primary activities with indole that surpass the benchmark TmTriple in lysate, with initial velocities of Trp formation up to 3-fold higher than WT-003-1-A (Fig. 3b and Supplementary Fig. 8) whose $k_{\text {cat }} / K_{\mathrm{M}}$ for indole is $1.37 \times 10^{5} \mathrm{M}^{-1}$ $\mathrm{s}^{-1}$, already 28 -fold higher than TmTriple's at saturating serine concentrations (Supplementary Table 4 and Supplementary Fig. 6). Second, direct comparison of some of the best panel variants to $T m$ Triple revealed dramatic general activity improvements for multiple indole analogs (Fig. 3b). For example, across the three most versatile variants (Q90*-003-1-C, Tri-003-1-D, and Q90*-003-1-D) the maximum fold-improvement in product yields over TmTriple were 37, 5, 19, and 50 using substrates 5$\mathrm{CN}, 7-\mathrm{CN}, 5-\mathrm{Br}$, and 6-Br, respectively (Fig. 3c). Finally, with the exception of 6-Br and azulene, at least one variant from the OrthoRep-evolved panel converted the indole analog substrates as well as or better than benchmark TrpBs Pf2B9, TmAzul, and Tm9D8*, which had been deliberately engineered toward new substrate scopes, though at higher temperatures (Fig. $3 \mathrm{~b}$ and Supplementary Fig. 9) $7,21,25,26$.

The diverse properties represented in our 63 variants were not just limited to primary activity increases on indole and promiscuous activities for indole analogs. Multiple variants from the panel also exhibited substantial improvements in selectivity for differently substituted indoles, which could be useful when working with substrate mixtures that may be less expensive to use industrially. For example, we observed many TmTrpBs with greater selectivity for $\mathbf{7 - B r}$ over $\mathbf{5 - B r}$ as compared to all four of the benchmark engineered TrpBs (Fig. 3d). Another variant in the panel, Tri-100-1-G, stood out for having appreciable activity with nearly all substrates tested, including $\mathbf{6}-\mathbf{C N}$ and $\mathbf{5}-\mathbf{C F}_{\mathbf{3}}$, which are poorly utilized by most other TrpBs, likely due to electron-withdrawing effects of their respective moieties. Notably, the ability to accept $\mathbf{5}-\mathbf{C F}_{\mathbf{3}}$ as a substrate was unique to Tri-100-1G: all other variants, as well as the benchmark TrpBs, showed no detectable product formation with this substrate (Fig. $3 \mathrm{e}$ and Supplementary Fig. 9). Repeating the reaction with purified enzyme in replicate confirmed the observed activity (Supplementary Fig. 10). Tri-100-1-G may therefore be a promising starting point for engineering efforts to access exotic Trp analogs. In short, despite having been selected for native activity with indole, OrthoRep-evolved TmTrpBs have extensive and diverse activities on a range of non-native substrates, demonstrating the value of depth and scale in the evolution of enzyme variants.

Mutations in evolved TmTrpBs may modulate conformational dynamics and fine tune the active site. Of the $\sim 200$ unique mutations in the OrthoRep-evolved $\operatorname{Tm} \operatorname{TrpBs}$ that we 

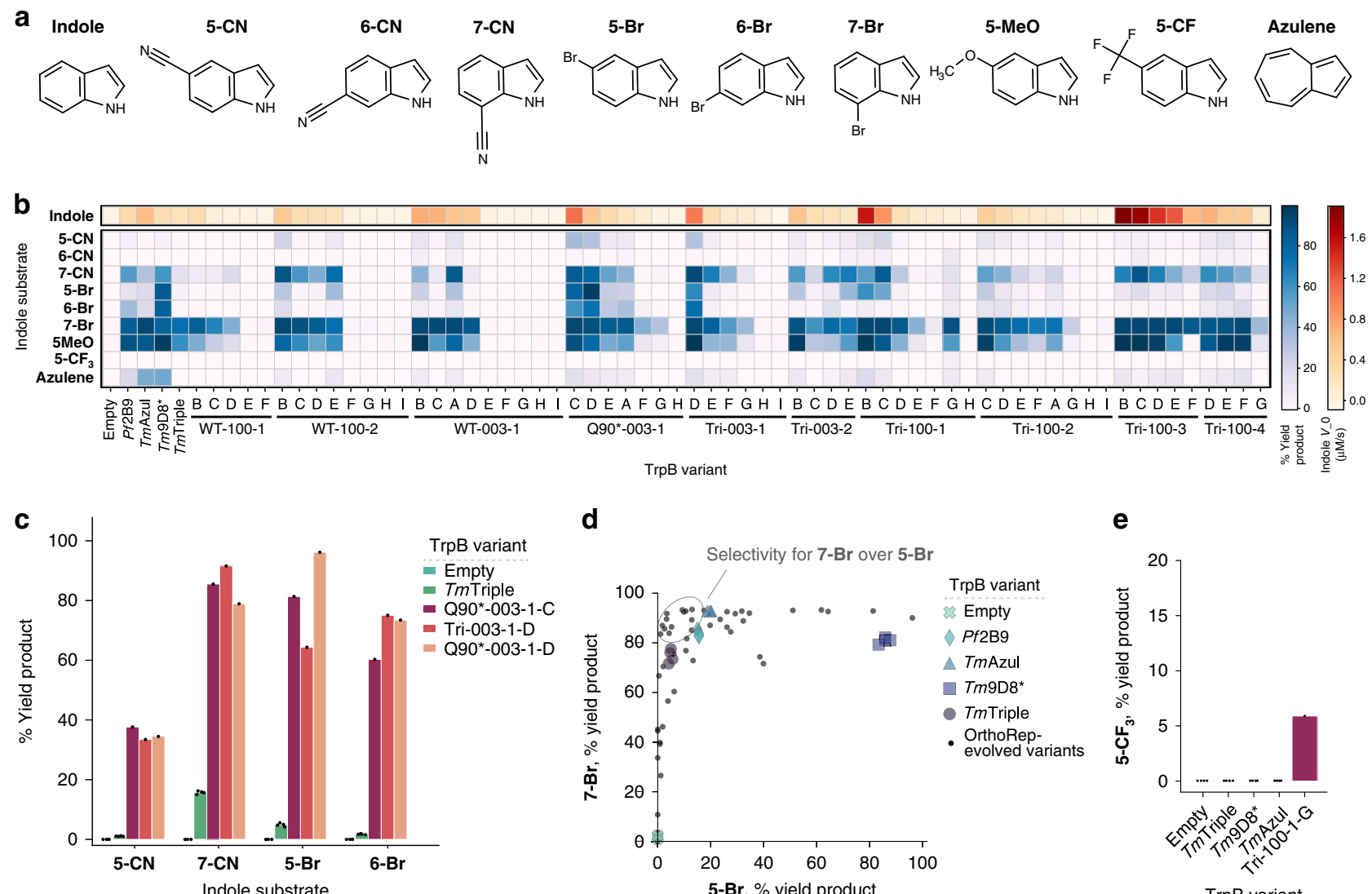

d

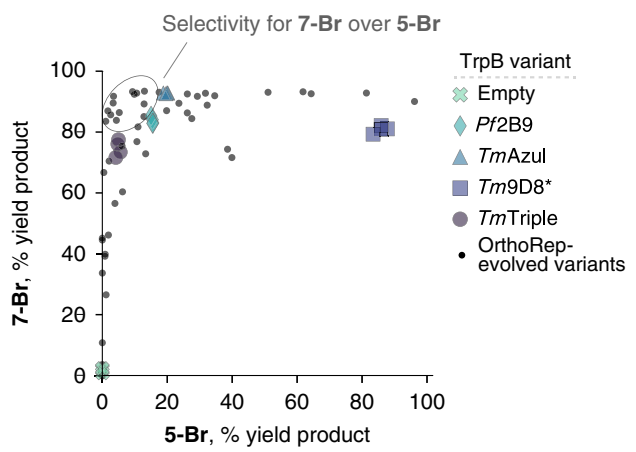

e

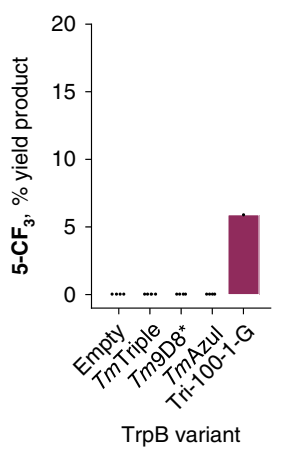

Fig. 3 Promiscuous activities of a panel of evolved TmTrpBs. a Indole substrates used to test the substrate scope of a panel of TmTrpB variants. 5-CN, 5-cyanoindole; 6-CN, 6-cyanoindole; 7-CN, 7-cyanoindole; 5-Br, 5-bromoindole; 6-Br, 6-bromoindole; 7-Br, 7-bromoindole; 5-MeO, 5-methoxyindole; $\mathbf{5}-\mathbf{C F}_{\mathbf{3}}, 5$-trifluoromethylindole. $\mathbf{b}$ Heatmap of TrpB activities reported as yield of the Trp analog produced from indicated substrates where $100 \%$ yield corresponds to full conversion of the indole analog to the Trp analog. Reactions were carried out using heat treated $\left(1 \mathrm{~h}\right.$ at $\left.75^{\circ} \mathrm{C}\right)$ cell lysate, yield was measured by HPLC-MS, and $V_{0}$ is the initial rate of Trp formation from indole at saturating serine concentrations. Panel TmTrpB variants are ordered first by the parental cultures from which they were derived, then by activity with indole. Reactions with OrthoRep-evolved variants other than WT-003-1-A, Q90*-003-1-A, and Tri-100-2-A were performed in one replicate and all other reactions performed in quadruplicate. Empty designates expression vector without any TrpB encoded. c Bar graph of selected indole analog activities from panel b. Points represent percentage HPLC yield for individual replicates, bars represent mean yield for multiple replicates or yield for a single replicate. d Activities of all variants shown in $\mathbf{b}$ for reactions with substrates 7-Br and 5-Br to show selectivity. Individual replicates are shown for empty vector and benchmark TrpBs and only mean values are shown for OrthoRep-evolved variants tested in replicate (i.e. WT-003-1-A, Q90*-003-1-A, and Tri-100-2-A) for clarity. e Heat treated lysate activity with $\mathbf{5}-\mathbf{C F}_{\mathbf{3}}$ for indicated TrpB variants. Source Data are provided with the Source data file.

characterized, there were some mutations whose effects could be rationalized from comparison to previous work. Since the TmTrpBs had to evolve stand-alone activity, it is unsurprising that many of the mutations we observed have been implicated in the loss of allosteric regulation by TrpA. For example, Buller et al. previously examined a series of engineered variants from Pyrococcus furiosus TrpB (PfTrpB) and found that evolution for stand-alone activity was facilitated by a progressive shift in the rate-limiting step from the first to the second stage of the catalytic cycle as well as stabilization of the 'closed' conformation of the enzyme ${ }^{27}$. That work implicated eight residues in this mechanism, seven of which correspond to homologous sites where we observed mutations in the evolved $\operatorname{Tm} \operatorname{TrpB}$ variants (i.e., P14, M18, I69, K96, L274, T292, and T321). Another mutation, N167D, present in three of the ten consensus sequences for evolved populations (Fig. 1c), has also been implicated in stabilizing the closed state ${ }^{21}$. Additional mutations observed but not studied before (e.g., S277F, S302P, and A321T) could also reasonably alter the allosteric network linking $\operatorname{Tm} \operatorname{TrpB}$ activity to its natural $T m \operatorname{TrpA}$ partner, based on existing structures and molecular dynamics simulations on the homologous Pf $\operatorname{TrpA} /$
PfTrpB complex ${ }^{22,27}$. Taken together, these mutations are likely implicated in converting allosteric activation by $\operatorname{Tm} \operatorname{TrpA}$ into constitutive activity to establish stand-alone function of TmTrpBs.

During the evolution of stand-alone activity, not only must allosteric activation by $\operatorname{Tm} \operatorname{TrpA}$ be recapitulated by mutations in $T m \operatorname{TrpB}$, the surface of $\operatorname{Tm} \operatorname{TrpB}$ that normally interacts with TmTrpA must adjust to a new local environment. Consistent with this adaptation, all consensus sequences for the ten successfully evolved populations from which our $\operatorname{Tm} \operatorname{TrpB}$ variants were sampled contain a mutation to at least one of a set of five residues located on the canonical TrpA interaction interface (Fig. 1c and Supplementary Fig. 11). These mutations might improve solubility by increasing hydrophilicity (e.g. G3D, Y8H, and A20T) or form intramolecular interactions that compensate for lost interactions with $\operatorname{Tm} \operatorname{TrpA}$, among other possibilities.

We also detected strong convergent evolution in a region near the catalytic lysine, K83, which directly participates in TmTrpB's catalytic cycle through covalent binding of PLP and multiple proton transfers (Supplementary Fig. 12) ${ }^{19}$. For example, A118 was mutated in the consensus sequence of four of the ten fully 
adapted populations, while adjacent residues T117 or A119 were mutated in an additional three (Fig. 1c). Furthermore, the three populations in which these residues were not mutated contained other consensus mutations that are either part of the $a$-helix to which K83 belongs, or, like residues 117-119, within $\sim 8 \AA$ of this helix (Fig. 1c and Supplementary Fig. 12). We hypothesize that the $a$-helix harboring K83 is a focal point of evolution, whereby mutations in its vicinity may finely adjust the positioning of K83 and the PLP cofactor to improve catalysis, perhaps as compensation for structural changes induced by thermoadaptation. Some OrthoRep-evolved variants also contained mutations to first- and second-shell active site residues (Supplementary Fig. 13), which may directly modulate the activity of $T m T r p B s$, although these mutations were rare. Taken together, we hypothesize that these mutations near the active site residues of TrpB were adaptive or compensatory.

The 20 mutations considered above are rationalized with respect to their impact on $\operatorname{Tm} \operatorname{TrpB}$ 's primary catalytic activity. While substrate promiscuity changes may be influenced by these explainable mutations, previous literature suggests that substrate specificity is globally encoded by amino acids distributed across an entire enzyme ${ }^{28}$. Indeed, the majority of the $\sim 200$ mutations found in our panel of TmTrpBs were far away from TmTrpB's active site and not rationalizable based on the known structural and kinetic properties of TrpBs. We suspect that the cryptic genetic variation this majority of mutations encompasses contributes to the diversity in substrate scope across our variants.

\section{Discussion}

In this work, we showed how the depth and scale of evolutionary search available in OrthoRep-driven protein evolution experiments could be applied to broaden the secondary promiscuous activities of $\operatorname{Tm} \operatorname{TrpB}$ while only selecting on its primary activity. The significance of this finding can be divided into two categories, one concerning the practical utility of the $\operatorname{Tm} \operatorname{TrpB}$ variants we obtained and the second concerning how this evolution strategy may apply to future enzyme evolution campaigns and protein engineering in general.

Practically, the TmTrpBs should find immediate use in the synthesis of Trp analogs. Trp analogs are valuable chiral precursors to pharmaceuticals as well as versatile molecular probes, but their chemical synthesis is challenged by stereoselectivity requirements and functional group incompatibility. This has spurred enzyme engineers to evolve TrpB variants capable of producing Trp analogs ${ }^{20,21,25,26}$, but the capabilities of available TrpBs are still limited. Compared to existing engineered TrpBs, our panel of variants has substantially higher activity for the synthesis of Trp and Trp analogs at moderate temperatures from almost all indole analogs tested and also accepts indole analogs, such as $\mathbf{5}-\mathbf{C F}_{\mathbf{3}}$ (Fig. 3a), for which benchmark TrpBs used in this study showed no detectable activity (Fig. 3e). (In fact, only one $\operatorname{TrpB}$ variant has shown detectable activity for this substrate in previous classical directed evolution campaigns ${ }^{21}$.) In addition, at least one member of the panel accepted each of the nine indole analogs we used to profile promiscuity, suggesting that additional indole analogs and non-indole nucleophiles not assayed here will also be accepted as substrates ${ }^{29,30}$. Finally, the evolved TmTrpBs are both thermostable and adapted for enzymatic activity at $30^{\circ} \mathrm{C}$. This maximizes their industrial utility, as thermostability predicts a protein's durability and can be exploited for simple heat-based purification processes, while mesophilic activity is compatible with heat-labile substrates, industrial processes where heating costs can compound, or in vivo applications in model mesophilic hosts (e.g. S. cerevisiae or E. coli).
Of more general significance may be the process through which the $\operatorname{Tm} \operatorname{TrpBs}$ in this study were generated. Previous directed evolution campaigns aiming to expand the substrate scope of TrpB screened directly for activity on indole analogs to guide the evolution process ${ }^{21,26}$, whereas this study only selected for TmTrpB's primary activity on indole. Yet this study still yielded $T m$ TrpBs whose secondary activities on indole analogs were both appreciable and diverse. Why?

A partial explanation may come from the high primary activities of OrthoRep-evolved TmTrpBs, as validated by kinetic measurements showing that variants tested have $k_{\mathrm{cat}} / K_{\mathrm{M}}$ values for indole well in the $10^{5} \mathrm{M}^{-1} \mathrm{~s}^{-1}$ range. Since OrthoRep drove the evolution of $\operatorname{Tm} \operatorname{TrpB}$ in a continuous format for $>100$ generations, each resulting $\operatorname{Tm} \operatorname{TrpB}$ is the outcome of many rounds of evolutionary improvement and change (evolutionary depth). This contrasts with previous directed evolution campaigns using only a small number of manual rounds of diversification and screening. Continuous OrthoRep evolution, on the other hand, allowed $\operatorname{Tm} \operatorname{TrpBs}$ to become quite catalytically efficient with minimal researcher effort. We suggest that the high primary catalytic efficiencies also elevated secondary activities of $\operatorname{Tm} \operatorname{TrpB}$, resulting in the efficient use of indole analogs. However, this explanation is not complete, as evolved $\operatorname{Tm}$ TrpBs with similar primary activity on indole had differences in secondary activities (Fig. 3). In other words, high primary activities did not uniformly raise some intrinsic set of secondary activities in $\operatorname{Tm} \operatorname{TrpB}$, but rather influenced if not augmented the secondary activities of $T m T r p B$ in different ways. We attribute this to the fact that we ran our evolution experiments in multiple independent replicates (evolutionary scale). Each replicate could therefore evolve the same primary activity through different mutational paths, the idiosyncrasies of which manifest as distinct secondary activities. A third explanation for the promiscuous profile diversity of these $\operatorname{Tm} \operatorname{TrpB}$ variants is that each replicate evolution experiment had, embedded within it, mechanisms to generate cryptic genetic variation without strong selection on primary activity. Many of the clones we sampled from each $\operatorname{Tm} \operatorname{TrpB}$ evolution experiment had altered promiscuity profiles but mediocre primary activity with indole (Fig. 3). We believe this is because OrthoRep drove TmTrpB evolution in the context of a multicopy plasmid such that non-neutral genetic drift from high activity sequences could occur within each cell at any given point. Therefore, $\operatorname{Tm} \operatorname{TrpB}$ sequences with fitness-lowering mutations could persist for short periods of time, potentially allowing for the crossing of fitness valleys during evolution experiments and, at the end of each evolution experiment, a broadening of the genetic diversity of clones even without explicitly imposed periods of relaxed selection. Since enzyme orthologs are capable of specializing towards different sets of secondary activities when pressured to do $\mathrm{so}^{2,3}$, non-neutral genetic drift from different consensus sequences across independent population should also access different secondary activities, further explaining the diversity of promiscuous activity profiles across clones selected from replicate evolution experiments. The combination of these mechanisms likely explains the variety of properties encompassed by the panel of TmTrpBs.

Our approach to $\operatorname{Tm} \operatorname{TrpB}$ evolution was inspired by the idea of gene orthologs in nature. Orthologs typically maintain their primary function while diversifying promiscuous activities through long evolutionary histories in different species ${ }^{2,31}$. We approximated this by evolving $\operatorname{Tm} \operatorname{TrpB}$ through continuous rounds of evolution, mimicking long histories, and in multiple replicates, mimicking the spatial separation and independence of species. Such depth and scale of evolutionary search is likely responsible for the substrate scope diversity of the $\operatorname{Tm} \operatorname{TrpBs}$ we report even as they were selected only on their primary activity. We recognize 

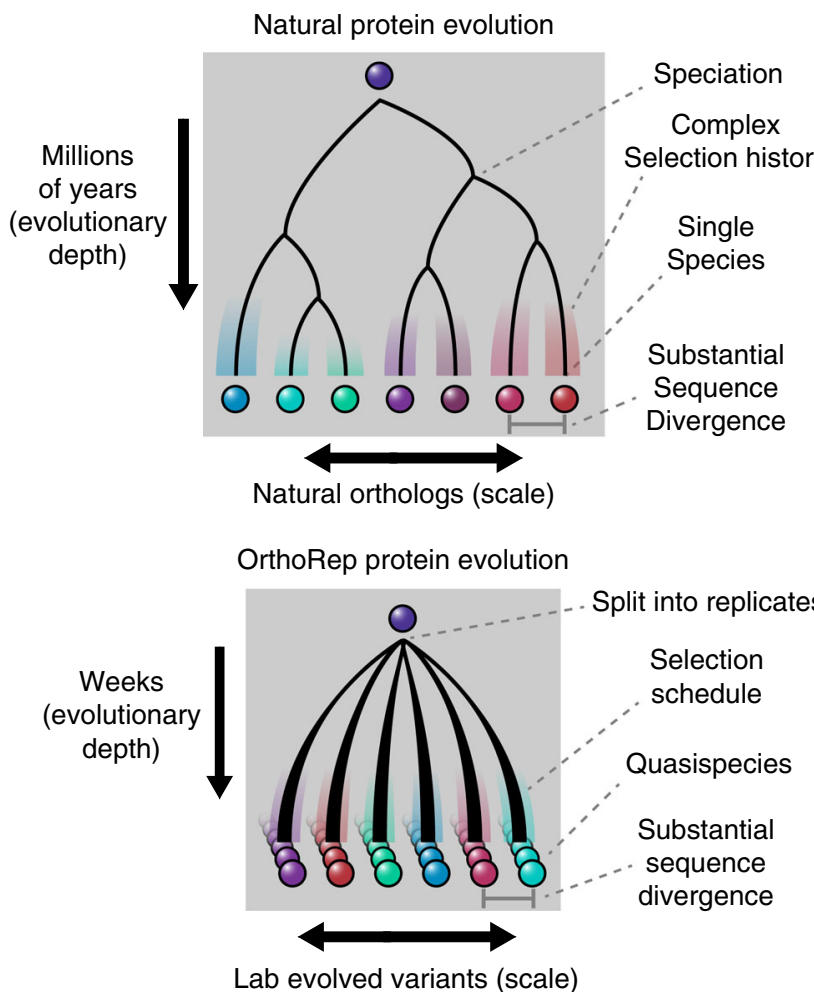

Fig. 4 Conceptual similarities between natural enzyme ortholog evolution and OrthoRep evolution. Splitting OrthoRep cultures into many replicates can be seen as a form of speciation occurring by spatial separation. Complex selection schedules may emulate varied selection histories of natural orthologs, generating substantial sequence divergence across replicates. Evolved OrthoRep cultures contain diverse populations of sequences akin to quasispecies owing to high mutation rates ${ }^{41}$.

that the evolved $\operatorname{Tm} \operatorname{TrpBs}$ represent lower diversity than natural orthologs. For example, the median amino acid sequence divergence between orthologous human and mouse proteins is $11 \%^{32}$, while the median divergence between pairs of variants from our experiment is $4.3 \%$ with a maximum of $8 \%$ (Supplementary Fig. 14). Still, this level of divergence between functional variants is substantial for a laboratory protein evolution experiment and suggests that it is realistic to model future work on the processes of natural ortholog evolution (Fig. 4). For example, it should be straightforward to scale our experiments further, to hundreds or thousands of independent populations each evolving over longer periods of time. This would better simulate the vastness of natural evolution. It should also be possible to deliberately vary selection schedules by adding competitive $\operatorname{Tm} \operatorname{TrpB}$ inhibitors (such as the very indole analogs for which they have promiscuous activity), changing temperatures, or cycling through periods of weak and strong selection at different rates. Such evolutionary courses would approximate complexity in natural evolutionary histories. These modifications to OrthoRep-driven $\operatorname{Tm} \operatorname{TrpB}$ evolution should yield greater cryptic genetic diversity, which may result in further broadening of promiscuous functions. The generation of cryptic genetic diversity at depth and scale should also be useful in efforts to predict protein folding and the functional effects of mutations via co-evolutionary analysis ${ }^{33,34}$. Indeed, catalogs of natural orthologs have proven highly effective in fueling such computational efforts, so our ability to mimic natural ortholog generation on laboratory timescales may be applicable to protein biology at large. Within the scope of enzyme engineering, we envision that the process of continuous replicate evolution, selecting only on primary activities of enzymes, will become a general strategy for expanding promiscuous activity ranges of enzymes as we and others extend it to new targets.

\section{Methods}

DNA plasmid construction. Plasmids used in this study are listed in Supplementary Data 1 . All plasmids that were not generated in a previous study were constructed via Gibson assembly ${ }^{35}$ from parts derived from the Yeast Toolkit ${ }^{24}$, from previously described OrthoRep integration cassette plasmids ${ }^{16}$, from E. coli expression vectors for previously described $\operatorname{TrpB}$ variants ${ }^{7,26}$, from synthesized oligonucleotides, from yeast genomic DNA, or from the standard E. coli expression vector, pET-22b(+). All DNA cloning steps and E. coli protein expression steps were performed in E. coli strains TOP10 and BL21(DE3), respectively. All oligonucleotides used for PCR were purchased from IDT and are described in Supplementary Data 2, and all enzymes and reagents used for cloning were purchased from NEB.

Parts used to generate yeast nuclear expression plasmids for testing the selection and p1 integration plasmids were PCR amplified from DNA sources listed above, Gibson assembled, transformed into E. coli, and plated onto selective LB agar plates. Individual clones were picked, grown to saturation in selective LB liquid media, miniprepped, and sequence confirmed. Following evolution of TrpB, individual variants were assembled into new yeast or $E$. coli expression vectors through PCR amplification of purified DNA from evolved yeast cultures, bulk cloning into the appropriate expression vector, picking individual colonies, and confirming absence of any frameshift mutations by Sanger sequencing.

Yeast strains and media. All yeast strains used in this study are listed in Sup plementary Data 3 . Yeast were incubated at $30^{\circ} \mathrm{C}$, with shaking at $200 \mathrm{rpm}$ for liquid cultures, and were typically grown in synthetic complete (SC) growth medium $(20 \mathrm{~g} / \mathrm{L}$ dextrose, $6.7 \mathrm{~g} / \mathrm{L}$ yeast nitrogen base w/o amino acids (US Biological), $2 \mathrm{~g} / \mathrm{L}$ SC dropout (US Biological) minus nutrients required for appropriate auxotrophy selection(s)), or were grown in YPD growth medium $(10 \mathrm{~g} / \mathrm{L}$ bacto yeast extract, $20 \mathrm{~g} / \mathrm{L}$ bacto peptone, $20 \mathrm{~g} / \mathrm{L}$ dextrose) with or without antibiotics, if no auxotrophic markers were being selected for. Media agar plates were made by combining $2 \mathrm{x}$ concentrate of molten agar and $2 \mathrm{x}$ concentrate of desired media formulation. Prior to all experiments, cells were grown to saturation in media selecting for maintenance of any plasmids present.

Yeast transformation. All yeast transformations were performed as described in Gietz and Shiest ${ }^{36}$. Briefly, a $4 \mathrm{~mL}$ culture of yeast was grown to mid-log phase in rich YPD medium (2\% (w/v) Bacto yeast extract, $4 \%(\mathrm{w} / \mathrm{v})$ Bacto peptone, $4 \%(\mathrm{w} / \mathrm{v})$ glucose) at $30^{\circ} \mathrm{C}$, harvested by centrifugation, washed with sterile water, and pelleted again by centrifugation. These pellets were then resuspended in a mixture containing PEG3350 (30\% (w/v) final concentration), lithium acetate ( $90 \mathrm{mM}$ final concentration), boiled salmon sperm carrier DNA $(0.25 \mathrm{mg} / \mathrm{mL}$ final concentration), and $\sim 1 \mu \mathrm{g}$ of the DNA to be transformed, all in a total volume of $410 \mu \mathrm{L}$. Transformations were often done in smaller scales where the described volumes were split into 8 transformations. Cells resuspended in the PEG3350/lithium acetate/DNA mixture were then incubated at $42^{\circ} \mathrm{C}$ for $30 \mathrm{~min}$., pelleted by centrifugation, resuspended in $1 \mathrm{~mL}$ YPD medium, incubated for $1 \mathrm{~h}$ at $30^{\circ} \mathrm{C}$ with shaking $(200 \mathrm{rpm})$, pelleted, resuspended in $1 \mathrm{~mL}$ sterile water, pelleted, and resuspended in an appropriate volume of sterile water or $0.9 \%(\mathrm{w} / \mathrm{v}) \mathrm{NaCl}$ for plating. Transformed cells were streaked onto selective media agar plates, and resulting single colonies were picked for all further uses. Transformations for integration onto p1 were performed as described previously ${ }^{15}: 2-4 \mu \mathrm{g}$ of plasmid DNA with ScaI restriction sites adjacent to integration flanks was cut with ScaI-HF (NEB) and transformed into yeast harboring the wt p1 and $\mathrm{p} 2$ plasmids. Proper integration was validated by miniprepping the resulting clonal strain, visualizing the recombinant $\mathrm{p} 1$ band of the desired size by gel electrophoresis of the miniprepped DNA, and PCR and Sanger sequencing of the gene of interest integrated onto pl.

To generate enough DNA for visualization of the recombinant p1 plasmid, high yield yeast minipreps were performed as previously described ${ }^{15}$. In brief, $1.5 \mathrm{~mL}$ of culture was pelleted, supernatant was discarded, and the pellet was resuspended in $1 \mathrm{~mL} 0.9 \% \mathrm{NaCl}$, pelleted again, and resuspended in $250 \mu \mathrm{L}$ Zymolyase solution (0.9 M D-Sorbitol (Sigma Aldrich), 0.1 M Ethylenediaminetetraacetic acid (EDTA, Sigma Aldrich), $10 \mathrm{U} / \mathrm{mL}$ Zymolyase (US Biological)). The suspension was incubated at $37^{\circ} \mathrm{C}$ for $1 \mathrm{~h}$, then centrifuged at $3000 \times g$ for $5 \mathrm{~min}$. The supernatant was discarded, and the pellet was resuspended in $280.5 \mu \mathrm{L}$ proteinase $\mathrm{K}$ solution ( $250 \mu \mathrm{L}$ TE (50 mM Tris-HCl (pH 7.5), 20 mM EDTA), $25 \mu \mathrm{L} 10 \%$ sodium dodecyl sulfate (SDS, Sigma Aldrich), $5.5 \mu \mathrm{L}$ proteinase K stock solution $(10 \mathrm{mg} / \mathrm{mL}$ proteinase K (Fisher) in water). Samples were then incubated at $65^{\circ} \mathrm{C}$ for $30 \mathrm{~min}$, combined with $75 \mu \mathrm{L} 5 \mathrm{M}$ potassium acetate (Fisher), and incubated on ice for 30 min. Samples were centrifuged at $12,000 \times g$ for $10 \mathrm{~min}$, the resulting supernatant was combined and mixed with $700 \mu \mathrm{L}$ ethanol (Gold Shield), and samples were then centrifuged at $3500 \times g$ for $15 \mathrm{~min}$. Supernatant was discarded and the resulting pellet was dried, resuspended in $150 \mu \mathrm{L}$ TE, and centrifuged at $12,000 \times g$ for $10 \mathrm{~min}$. Supernatant was then combined with $8 \mu \mathrm{L} 1 \mathrm{mg} / \mathrm{mL}$ ribonuclease A (Thermo Scientific) and incubated at $37^{\circ} \mathrm{C}$ for $30 \mathrm{~min}$, combined with $150 \mu \mathrm{L}$ 
isopropanol (Fisher), and centrifuged at $12,000 \mathrm{~g}$ for $10 \mathrm{~min}$ to pellet purified DNA. Pellet is dried, then resuspended in $30 \mu \mathrm{L}$ water.

Following confirmation of the presence of the desired recombinant $\mathrm{p} 1$, strains were then transformed with either of two plasmids for nuclear expression of an OrthoRep terminal protein DNA polymerase 1 (TP-DNAP1) variant: wt TPDNAP1 (pAR-Ec318) for evaluating trp5 complementation of TrpB variants without mutagenesis, or error-prone TP-DNAP1 (pAR-Ec633) for generating strains ready for TrpB evolution. These strains were passaged for $\sim 40$ generations to stabilize copy number of the recombinant $\mathrm{p} 1$ species, prior to any use in experiments.

Genomic deletion of the entire TRP5 ORF was accomplished through cotransformation of a CRISPR/Cas9 plasmid targeting TRP5 with the spacer sequence TTTGAGCCTGATCCCACTAG and a linear DNA fragment comprised of two concatenated $50 \mathrm{bp}$ homology flanks to the TRP $5 \mathrm{ORF}^{37}$. Transformations were then plated on selective media agar, colonies were re-streaked onto nonselective media agar, and resulting colonies were grown to saturation in liquid media. The region of interest was PCR amplified and Sanger sequenced to confirm presence of desired modification.

Plating assays. Yeast strains expressing a $\operatorname{TrpB}$ variant either from a nuclear plasmid, or from p1 with wt OrthoRep polymerase (TP-DNAP1) expressed from a nuclear plasmid, were grown to saturation in SC $-\mathrm{L}$ or SC $-\mathrm{LH}$, spun down, washed once with $0.9 \% \mathrm{NaCl}$, then spun down again, and the resulting pellet was resuspended in $0.9 \% \mathrm{NaCl}$. Washed cells were then diluted 1:100 (or 1:10,000 where indicated) in $0.9 \% \mathrm{NaCl}$, and $10 \mu \mathrm{L}$ of each diluted cell suspension was plated onto media agar plates in pre-marked positions. After 3 days of growth, cell spots were imaged (Bio-Rad ChemiDoc $\left.{ }^{\mathrm{mm}}\right)$. Resulting images were adjusted uniformly ("High" set to 40,000) to improve visibility of growth (Bio-Rad Image Lab Software). Figures utilizing these images (Supplementary Figs. 1 and 2) were made by manually combining images of different plates, but all images of the same media condition within each figure panel were derived from the same image of a single plate.

TmTrpB evolution. Yeast strains with a nuclear plasmid expressing error-prone TP-DNAP1 and with wt TmTrpB, Tm Triple, or TmTripleQ90* encoded on p1 (GR-Y053, GR-Y055, and GR-Y057, Supplementary Data 3) were grown to saturation in $\mathrm{SC}-\mathrm{LH}$, prior to passaging for evolution. All cultures passaged for evolution of $\operatorname{Tm} \operatorname{TrpB}$ regardless of success are described in Supplementary Table 1. To provide enough indole substrate for sufficient Trp production, but not enough to induce toxicity, all growth media used for evolution of $\operatorname{TrpB}$ activity was supplemented with $100 \mu \mathrm{M}$ indole, as informed by results shown in Supplementary Fig. 1. All passages for evolution were carried out as 1:100 dilutions. To induce a growth defect but still allow for some growth, the first passage for each evolution culture was carried out in SC - LH media with $37 \mu \mathrm{M} \operatorname{Trp}(7.6 \mathrm{mg} / \mathrm{L})$. After two or three days of shaking incubation, if $\mathrm{OD}_{600}>1.0$ (Bio-Rad SmartSpec ${ }^{\mathrm{mI}}$ 3000) for $100 \mathrm{~mL}$ cultures, or if most wells in a 24 well block of $3 \mathrm{~mL}$ cultures were saturated to a similar degree by eye, cultures were passaged into fresh growth medium with a slightly reduced Trp concentration. If the level of growth was beneath this threshold, the culture was passaged into growth medium with the same Trp concentration. This process was continued until cultures were capable of growth in a Trp concentration of $3.7 \mu \mathrm{M}$ (or, in the sole case of WT-100-1, 4.7 $\mu \mathrm{M}$ ), at which point a passage into media lacking Trp was attempted, which typically resulted in successful growth. Resulting cultures were then passaged six additional times into growth medium lacking Trp.

Growth rate assays. Yeast strains containing nuclear plasmids encoding one of several OrthoRep-evolved TrpB variants, wt $\operatorname{Tm} \operatorname{TrpB}$, TmTriple, or none of these (denoted "empty") were grown to saturation in SC -L, washed as described above, then inoculated 1:100 into multiple media conditions in 96-well clear-bottom plates, with four biological replicates per media/strain combination. Plates were then sealed with a porous membrane and allowed to incubate with shaking at $30^{\circ} \mathrm{C}$ for $24 \mathrm{~h}$, with $\mathrm{OD}_{600}$ measurements taken automatically every $30 \mathrm{~min}$ (Tecan Infinite M200 Pro), according to a previously described protocol ${ }^{38}$. Multiple $24 \mathrm{~h}$ periods were required for each experiment, but empty controls were included in each individual 96-well plate to ensure validity of growth in other cultures. Raw $\mathrm{OD}_{600}$ measurements were fed into a custom MATLAB script ${ }^{18}$, which carries out a logarithmic transformation to linearize the exponential growth phase, identifies this growth phase, and uses this to calculate the doubling time (T). Doubling time was then converted to growth rate (gr) using equation (1):

$$
g r=\frac{\ln (2)}{T} .
$$

Enzyme characterization. Chemicals and reagents were purchased from commercial sources and used without further purification. All cultures were grown in Terrific Broth supplemented with $100 \mu \mathrm{g} / \mathrm{mL}$ carbenicillin $\left(\mathrm{TB}_{\mathrm{carb}}\right)$. Cultures were shaken in a New Brunswick Innova 4000 (shaking diameter $19 \mathrm{~mm}$ ), with the exception of the 96-well deep-well plates (USA Scientific), which were shaken in a Multitron INFORS HT (shaking diameter $50 \mathrm{~mm}$ ). Lysis buffer was composed of
$50 \mathrm{mM}$ potassium phosphate, $\mathrm{pH} 8.0$ (KPi buffer), supplemented with 100 or 200 $\mu \mathrm{M}$ pyridoxal $5^{\prime}$-phosphate (PLP). Heat lysis was performed in a $75{ }^{\circ} \mathrm{C}$ water bath (Fisher) for $>1 \mathrm{~h}$. Protein concentrations were determined using a Pierce $\mathrm{e}^{\mathrm{m}} \mathrm{BCA}$ Protein Assay Kit (Thermo Scientific). Reactions were performed in KPi buffer. Liquid chromatography/mass spectrometry (LCMS) was performed on an Agilent 1290 UPLC-LCMS equipped with a C-18 silica column $(1.8 \mu \mathrm{m}, 2.1 \times 50 \mathrm{~mm})$ using $\mathrm{CH}_{3} \mathrm{CN} / \mathrm{H}_{2} \mathrm{O}\left(0.1 \%\right.$ acetic acid by volume): $5-95 \% \mathrm{CH}_{3} \mathrm{CN}$ over $2 \mathrm{~min} ; 1 \mathrm{~mL}$ min. Liquid chromatography/mass spectrometry (LCMS) was also performed on an Agilent 1260 HPLC-MS equipped with Agilent InfinityLab Poroshell 120 ECC18 column $(2.7 \mu \mathrm{m}, 4.6 \times 50 \mathrm{~mm})$ : hold $5 \% \mathrm{CH}_{3} \mathrm{CN}$ for $0.5 \mathrm{~min}, 5-95 \% \mathrm{CH}_{3} \mathrm{CN}$ over $2 \mathrm{~min} ; 1 \mathrm{~mL} / \mathrm{min}$.

TrpB variants selected for further characterization were cloned into a pET-22b $(+)$ vector with a C-terminal 6X His-tag and transformed into E. coli BL21(DE3) cells (Lucigen)

Large scale expression and lysis. A single colony containing the appropriate $\operatorname{TrpB}$ gene was used to inoculate $5 \mathrm{~mL} \mathrm{~TB}$ carb and incubated overnight at $37^{\circ} \mathrm{C}$ and $230 \mathrm{rpm}$. For expression, $0.5 \mathrm{~mL}$ of overnight culture were used to inoculate $50 \mathrm{~mL}$ $\mathrm{TB}_{\text {carb }}$ in a $250 \mathrm{~mL}$ flask and incubated at $37^{\circ} \mathrm{C}$ and $250 \mathrm{rpm}$ for $3 \mathrm{~h}$ to reach $\mathrm{OD}_{600}$ $0.6-0.8$. Cultures were chilled on ice for $20 \mathrm{~min}$ and expression was induced with a final concentration of $1 \mathrm{mM}$ isopropyl $\beta$-d-thiogalactopyranoside (IPTG).

Expression proceeded at $25^{\circ} \mathrm{C}$ and $250 \mathrm{rpm}$ for $\sim 20 \mathrm{~h}$. Cells were harvested by centrifugation at $5000 \mathrm{~g}$ for $5 \mathrm{~min}$ at $4^{\circ} \mathrm{C}$, and then the supernatant was decanted. The pellet was stored at $-20^{\circ} \mathrm{C}$ until further use or used immediately for whole cell transformations.

Pellets were lysed in $5 \mathrm{~mL}$ of lysis KPi buffer with $200 \mu \mathrm{M}$ PLP, supplemented with $1 \mathrm{mg} / \mathrm{mL}$ lysozyme (HEWL, Sigma Aldrich), $0.02 \mathrm{mg} / \mathrm{mL}$ bovine pancreas DNase I, and 0.1x BugBuster (Novagen) and incubated at $37^{\circ} \mathrm{C}$ for $30 \mathrm{~min}$. Lysate was clarified by centrifugation at $5,000 \mathrm{~g}$ for $10 \mathrm{~min}$, divided into $1 \mathrm{~mL}$ aliquots, and stored at $-20^{\circ} \mathrm{C}$ until further use.

Lysate and whole cell small-scale reactions. Protein concentration in lysate was quantified by BCA. Lysate reactions were performed in $2 \mathrm{~mL}$ glass HPLC vials (Agilent) charged with indole (final conc. $20 \mathrm{mM}$ ) dissolved in DMSO (5\% w/v), followed by the addition of lysate (final enzyme conc. $4 \mu \mathrm{M}$ ), and serine (final conc. $20 \mathrm{mM}$ ) to achieve a final volume of $200 \mu \mathrm{L}$. Whole cell reactions were performed in $2 \mathrm{~mL}$ glass HPLC vials (Agilent) charged with indole (final conc. $20 \mathrm{mM}$ ) dissolved in DMSO (5\% w/v), followed by the addition of cells diluted in KPi buffer (final $\mathrm{OD}_{600}=6$ ), and serine (final conc. $20 \mathrm{mM}$ ) to achieve a final volume of $200 \mu \mathrm{L}$. Reactions were incubated at $30^{\circ} \mathrm{C}$ for $24 \mathrm{~h}$, diluted with $800 \mu \mathrm{L} 1: 1$ $\mathrm{CH}_{3} \mathrm{CN} / 1 \mathrm{M}$ aq. $\mathrm{HCl}$, and analyzed via UHPLC-MS.

Thermostability determination. Enzyme $\mathrm{T}_{50}$ measurements (the temperature at which $50 \%$ of the enzyme is irreversibly inactivated after a $1 \mathrm{~h}$ incubation) were used to report on the thermostability of the enzyme. In a total volume of $100 \mu \mathrm{L}$, samples were prepared in KPi buffer with $1 \mu \mathrm{M}$ enzyme in PCR tubes and either set aside $\left(25^{\circ} \mathrm{C}\right)$ or heated in a thermal cycler on a gradient from $79-99^{\circ} \mathrm{C}$ (OrthoRepgenerated variants), or $59-99^{\circ} \mathrm{C}$ (TmTriple), for $1 \mathrm{~h}$, with each temperature performed in duplicate. Precipitated protein was pelleted via centrifugation and $75 \mu \mathrm{L}$ of each sample was carefully removed and added to the wells of a 96-well UVtransparent assay plate containing $0.5 \mathrm{mM}$ indole and $0.5 \mathrm{mM}$ serine. Relative product formation was observed by measuring the change in absorbance at $290 \mathrm{~nm}$ to determine the temperature at which the sample had $50 \%$ residual activity compared to the $25^{\circ} \mathrm{C}$ samples (modeled as a logistic function).

Enzyme kinetics. Enzymatic parameters, $k_{\mathrm{cat}}$ and $K_{\mathrm{M}}$, for the conversion of indole to Trp were estimated via Bayesian inference assuming Michaelis-Menten behavior under saturating serine $(40 \mathrm{mM})$ in KPi buffer. Briefly, initial velocities $(v)$ were determined by monitoring Trp formation in a Shimadzu UV-1800 spectrophotometer at $30^{\circ} \mathrm{C}$ for 1 min over a range of indole concentrations at $290 \mathrm{~nm}$ using the reported indole-Trp difference in absorbance coefficient $\left(\Delta \varepsilon_{290}=\right.$ $\left.1.89 \mathrm{mM}^{-1} \mathrm{~cm}^{-1}\right)^{39}$. These velocities were modeled using equation (2):

$$
v=\frac{\left.V_{\max } \text { [indole }\right]}{K_{\mathrm{M}}+[\text { indole }]}
$$

and estimates for $v$ and $V_{\max }$ were converted to $k$ and $k_{\text {cat }}$ by normalizing for enzyme concentration. Parameter estimates are obtained as Hamiltonian Markov chain Monte Carlo (MCMC) posterior samples and reported as the median with their 95\% credible regions (CR). The MCMC software used for sampling was Stan (pystan version 2.19.0.0). The sampling was performed with four separate chains, each starting with 2000 warm-up (disregarded) steps followed by 12000 posterior sampling steps. The priors chosen for $k_{\text {cat }}$ and $K_{\mathrm{M}}$ were lognormal distributions with means $\log (150)$ and $\log (500)$, standard deviations 2.5 and 1.5 , with units of $\sec ^{-1}$ and $\mu \mathrm{M}$, respectively. This provided non-negative probability density that covered low-to-moderate values of the parameters for many known enzymes, but still had significant density out to very high values for each parameter. (In all cases, the data was shown to significantly inform the prior.) The code used to generate these estimates (along with example data) can be found at http://github.com/ palmhjell/bayesian_kinetics. 
Table 1 Sources and reaction concentrations for indole analogs.

\begin{tabular}{lllll} 
Nucleophile & Source & Catalog \# & CAS & Concentration (mM) \\
\hline 5-Cyanoindole & Chem Impex & 21849 & $15861-24-2$ & 20 \\
6-Cyanoindole & Chem Impex & 21181 & $15861-36-6$ & 20 \\
7-Cyanoindole & Sigma & CDSO08484 & $10075-50-0$ & 20 \\
5-Bromoindole & Sigma & B68607 & $52415-29-9$ & 20 \\
6-Bromoindole & Sigma & 524344 & $51417-51-7$ & 20 \\
7-Bromoindole & Sigma & 473723 & $1006-94-6$ & 20 \\
5-Methoxyindole & Combi-Blocks & IN0049 & $100846-24-0$ & 20 \\
5-Trifluoromethylindole & Sigma & 701068 & $275-51-4$ & 10 \\
Azulene & Alfa Aesar & L08271 & \\
\hline
\end{tabular}

Small-scale expression and lysis. Variants were arrayed into a 96-well deep-well plate along with $T m$ Triple, Tm9D8*, TmAzul, and $P f 2 B$. Individual colonies were grown in $600 \mu \mathrm{L} \mathrm{TB}_{\text {carb }}$ in 96-well polypropylene plates overnight at $37^{\circ} \mathrm{C}$, $250 \mathrm{rpm}, 80 \%$ humidity. The following day, $20 \mu \mathrm{L}$ of overnight culture was used to inoculate $630 \mu \mathrm{L} \mathrm{TB}$ carb in deep-well 96-well plates and grown at $37^{\circ} \mathrm{C}, 250 \mathrm{rpm}$. After $4 \mathrm{~h}$, cultures were chilled on ice for 20-30 min and protein expression was induced with $50 \mu \mathrm{L}$ IPTG (final conc. $1 \mathrm{mM}$ ) diluted in $\mathrm{TB}_{\text {carb. }}$. Cultures were shaken at $20^{\circ} \mathrm{C}, 250 \mathrm{rpm}$ for $20-24 \mathrm{~h}$, after which they were subjected to centrifugation at $5,000 \mathrm{~g}$ for $10 \mathrm{~min}$. The cell pellets were frozen at $-20^{\circ} \mathrm{C}$ until further use or used immediately.

Indole rate measurements. Pellets were lysed in either $600 \mu \mathrm{L}$ of KPi buffer with $100 \mu \mathrm{M}$ PLP and heat treated at $75^{\circ} \mathrm{C}$ for $1 \mathrm{~h}$, or in $600 \mu \mathrm{L}$ of this buffer supplemented with $1 \mathrm{mg} / \mathrm{mL}$ lysozyme, $0.02 \mathrm{mg} / \mathrm{mL}$ bovine pancreas DNase I, and $0.1 \mathrm{x}$ BugBuster and incubated at $37^{\circ} \mathrm{C}$ for $1 \mathrm{~h}$. Lysate from both conditions was clarified by centrifugation at $4500 \mathrm{~g}$ for $10 \mathrm{~min}$ and stored at $4{ }^{\circ} \mathrm{C}$ until further use.

Reaction master mix composed of $625 \mu \mathrm{M}$ indole and $25 \mathrm{mM}$ serine in $\mathrm{KPi}$ buffer was prepared and, before reactions, plates and master mix were incubated in $30^{\circ} \mathrm{C}$ water bath for $30 \mathrm{~min}$. The microplate reader (Tecan Spark) was also preheated to $30^{\circ} \mathrm{C}$.

To UV-transparent 96-well assay plates (Caplugs, catalog \# 290-8120-0AF), $160 \mu \mathrm{L}$ pre-heated reaction master mix was added by 12 -channel pipet followed by $40 \mu \mathrm{L}$ of lysate from the pre-heated plate using a Microlab NIMBUS96 liquid handler (Hamilton). Plates were immediately transferred into the plate reader, shaken for $10 \mathrm{sec}$ to mix and the absorbance of each well at $290 \mathrm{~nm}$ was recorded as rapidly as possible ( $\sim 20 \mathrm{sec}$ between measurements) for 120 cycles. The rate of product formation was determined by finding the rate of absorbance change over time and converting to units of concentration using $\Delta \varepsilon_{290}=1.89 \mathrm{mM}^{-1} \mathrm{~cm}^{-1}$ (see above) and a determined path length of $0.56 \mathrm{~cm}$. We observed no systematic difference in activity between the two lysate preparations (Supplementary Fig. 15), suggesting that most enzyme variants retained sufficient thermostability for purification via heat treatment, and this method was used in subsequent experiments.

Substrate scope screen. Pellets were lysed in $300 \mu \mathrm{L}$ KPi buffer with $200 \mu \mathrm{M}$ PLP and clarified by centrifugation at $4000 \times g$ for $10 \mathrm{~min}$. To a 96 -well deep-well plate charged with $10 \mu \mathrm{L}$ nucleophile dissolved in DMSO (See Table 1 for final reaction concentrations), $40 \mu \mathrm{L}$ of the heat treated lysate was transferred using a Microlab NIMBUS96 liquid handler (Hamilton), followed by addition of $150 \mu \mathrm{L}$ serine (final conc. $20 \mathrm{mM}$ ) with a 12-channel pipet. Reactions were sealed with 96-well ArctiSeal ${ }^{\text {Tw }}$ Silicone/PTFE Coating (Arctic White) and incubated in $30^{\circ} \mathrm{C}$ water bath for $\sim 24 \mathrm{~h}$. Reactions were diluted with $600 \mu \mathrm{L} 2: 1 \mathrm{CH}_{3} \mathrm{CN} / 1 \mathrm{M}$ aq. $\mathrm{HCl}$, subjected to centrifugation at $5000 \mathrm{~g}$, and $400 \mu \mathrm{L}$ was transferred to $2 \mathrm{~mL}$ glass HPLC vials (Agilent). Samples were analyzed by HPLC-MS. Azulene samples were further diluted 20x to avoid oversaturation of the UV-detector and analyzed via UHPLCMS.

All samples except those containing azulene were analyzed at $277 \mathrm{~nm}$, representing the isosbestic point between indole and Trp and allowing estimation of yield by comparing the substrate and product peak areas for indole analogs ${ }^{21}$. Azulene yield was estimated as described previously ${ }^{25}$. Nucleophile retention times were determined though injection of authentic standards and product retention times were identified by extracting their expected mass from the mass spectrum.

\section{Large-scale expression and purification of Tri-100-3-F and Tri-100-1-G. A} single colony containing the appropriate $\operatorname{TrpB}$ gene was used to inoculate $5 \mathrm{~mL}$ $\mathrm{TB}_{\text {carb }}$ and incubated overnight at $37^{\circ} \mathrm{C}$ and $230 \mathrm{rpm}$. For expression, $2.5 \mathrm{~mL}$ of overnight culture were used to inoculate $250 \mathrm{~mL} \mathrm{~TB}_{\mathrm{carb}}$ in a 1-L flask and incubated at $37^{\circ} \mathrm{C}$ and $250 \mathrm{rpm}$ for $3 \mathrm{~h}$ to reach $\mathrm{OD}_{600} 0.6-0.8$. Cultures were chilled on ice for $20 \mathrm{~min}$ and expression was induced with a final concentration of $1 \mathrm{mM}$ IPTG. Expression proceeded at $25^{\circ} \mathrm{C}$ and $250 \mathrm{rpm}$ for $\sim 20 \mathrm{~h}$. Cells were harvested by centrifugation at $5000 \mathrm{~g}$ for $5 \mathrm{~min}$ at $4^{\circ} \mathrm{C}$, and then the supernatant was decanted. The pellet was stored at $-20^{\circ} \mathrm{C}$ until further use.
Pellets were lysed in $25 \mathrm{~mL} \mathrm{KPi}$ buffer with $200 \mu \mathrm{M}$ PLP for $>1 \mathrm{~h}$ at $75^{\circ} \mathrm{C}$. Lysate was clarified by spinning $14,000 \mathrm{~g}$ for $20 \mathrm{~min}$ at $4^{\circ} \mathrm{C}$ (New Brunswick Avanti $\mathrm{J}-30 \mathrm{I}$ ). Protein was purified over hand-packed HisPur ${ }^{\mathrm{mm}} \mathrm{Ni}$-NTA Resin (Thermo Scientific, catalog \# 88221), dialyzed into KPi buffer and quantified by BCA.

Tri-100-3-F PLP-binding assay. Variant Tri-100-3-F did not the exhibit characteristic yellow color of PLP-bound TrpB variants after purification, however BCA indicated comparable protein concentrations to the Tri-100-1-G variant. We have previously observed that some $\operatorname{TrpB}$ variants lose binding affinity for PLP resulting in non-functional apoenzyme. We evaluated Trp formation of Tri-100-3-F supplemented with $0,0.1,0.25,0.5,1,2,5$, and $100 \mu \mathrm{M}$ PLP via UV-Vis spectrophotometry. Serine (final conc. $25 \mathrm{mM}$ ) + PLP master mixes of the eight concentrations were prepared and dispensed into 96-well UV-transparent plate. Enzyme (final conc. $1 \mu \mathrm{M}$ ) with or without indole master mixes were prepared and $100 \mu \mathrm{L}$ dispensed into 96 -well plate. The plate was immediately transferred into plate reader, shaken for $10 \mathrm{~s}$ to mix and product formation was measured $\sim 20 \mathrm{sec}$ for 120 cycles at $290 \mathrm{~nm}$.

Only the $100 \mu \mathrm{M}$ condition restored activity, supporting our hypothesis that the purified enzyme was apoprotein and binds PLP poorly, requiring supplementation of PLP to re-form a functional holoenzyme. Thus, we chose to supplement PLP in the subsequent purified protein reactions.

Tri-100-3-F and Tri-100-1-G small-scale analytical reactions. Reactions were performed in $2 \mathrm{~mL}$ glass HPLC vials (Agilent) charged with nucleophile (final conc. $20 \mathrm{mM})$ dissolved in DMSO $(5 \% \mathrm{w} / \mathrm{v})$, followed by the addition of purified protein (final enzyme conc. either $2 \mu \mathrm{M}$ or $40 \mu \mathrm{M}$ ), PLP (final conc. $100 \mu \mathrm{M}$ ) and serine (final conc. $20 \mathrm{mM}$ ) to achieve a final volume of $200 \mu \mathrm{L}$. Reactions were incubated at $30^{\circ} \mathrm{C}$ for $\sim 24 \mathrm{~h}$. Reactions were diluted with $600 \mu \mathrm{L} 2: 1 \mathrm{CH}_{3} \mathrm{CN} / 1 \mathrm{M}$ aq. $\mathrm{HCl}$, subjected to centrifugation at $5000 \times g$, and $400 \mu \mathrm{L}$ transferred to $2 \mathrm{~mL}$ glass HPLC vials (Agilent). Samples were analyzed by HPLC-MS. Azulene samples were further diluted 20x to avoid oversaturation of the UV-detector and analyzed via UHPLC-MS.

Reporting summary. Further information on research design is available in the Nature Research Reporting Summary linked to this article.

\section{Data availability}

The datasets generated and/or analyzed during the current study are available from the corresponding author upon request. Source data are provided with this paper.

\section{Code availability}

The code used to generate enzyme kinetic estimates (along with example data) can be found at http://github.com/palmhjell/bayesian_kinetics.

Received: 24 June 2020; Accepted: 19 October 2020; Published online: 06 November 2020

\section{References}

1. Tatusov, R. L., Koonin, E. V. \& Lipman, D. J. A genomic perspective on protein families. Science 278, 631-637 (1997).

2. Khanal, A., McLoughlin, S. Y., Kershner, J. P. \& Copley, S. D. Differential effects of a mutation on the normal and promiscuous activities of orthologs: implications for natural and directed evolution. Mol. Biol. Evol. 32, 100-108 (2015).

3. Baier, F. et al. Cryptic genetic variation shapes the adaptive evolutionary potential of enzymes. Elife 8, 1-20 (2019).

4. Aharoni, A. et al. The 'evolvability' of promiscuous protein functions. Nat. Genet. 37, 73-76 (2005). 
5. Khersonsky, O. \& Tawfik, D. S. Enzyme promiscuity: a mechanistic and evolutionary perspective. Annu. Rev. Biochem. 79, 471-505 (2010).

6. Leveson-Gower, R. B., Mayer, C. \& Roelfes, G. The importance of catalytic promiscuity for enzyme design and evolution. Nat. Rev. Chem. 3, 687-705 (2019).

7. Murciano-Calles, J., Romney, D. K., Brinkmann-Chen, S., Buller, A. R. \& Arnold, F. H. A panel of TrpB biocatalysts derived from tryptophan synthase through the transfer of mutations that mimic allosteric activation. Angew. Chem. Int. Ed. 55, 11577-11581 (2016).

8. Devine, P. N. et al. Extending the application of biocatalysis to meet the challenges of drug development. Nat. Rev. Chem. 2, 409-421 (2018).

9. Truppo, M. D. Biocatalysis in the pharmaceutical industry: the need for speed. ACS Med. Chem. Lett. 8, 476-480 (2017).

10. Almhjell, P. J., Boville, C. E. \& Arnold, F. H. Engineering enzymes for noncanonical amino acid synthesis. Chem. Soc. Rev. 47, 8980-8997 (2018).

11. Zheng, J., Payne, J. L. \& Wagner, A. Cryptic genetic variation accelerates evolution by opening access to diverse adaptive peaks. Science 365, 347-353 (2019).

12. Gupta, R. D. \& Tawfik, D. S. Directed enzyme evolution via small and effective neutral drift libraries. Nat. Methods 5, 939-942 (2008).

13. Bloom, J. D., Romero, P. A., Lu, Z. \& Arnold, F. H. Neutral genetic drift can alter promiscuous protein functions, potentially aiding functional evolution. Biol. Direct 2, 7-10 (2007).

14. Bershtein, S., Goldin, K. \& Tawfik, D. S. Intense neutral drifts yield robust and evolvable consensus proteins. J. Mol. Biol. 379, 1029-1044 (2008).

15. Ravikumar, A., Arrieta, A. \& Liu, C. C. An orthogonal DNA replication system in yeast. Nat. Chem. Biol. 10, 175-177 (2014).

16. Ravikumar, A., Arzumanyan, G. A., Obadi, M. K. A., Javanpour, A. A. \& Liu, C. C. Scalable, continuous evolution of genes at mutation rates above genomic error thresholds. Cell 175, 1946-1957.e13 (2018).

17. Packer, M. S. \& Liu, D. R. Methods for the directed evolution of proteins. Nat. Rev. Genet. 16, 379-394 (2015).

18. Zhong, Z. et al. Automated continuous evolution of proteins in vivo. ACS Synth. Biol. https://doi.org/10.1021/acssynbio.0c00135 (2020).

19. Dunn, M. F. Allosteric regulation of substrate channeling and catalysis in the tryptophan synthase bienzyme complex. Arch. Biochem. Biophys. 519 154-166 (2012).

20. Buller, A. R. et al. Directed evolution of the tryptophan synthase $\beta$-subunit for stand-alone function recapitulates allosteric activation. Proc. Natl Acad. Sci. 112, 14599-14604 (2015)

21. Romney, D. K., Murciano-Calles, J., Wehrmüller, J. E. \& Arnold, F. H. Unlocking reactivity of TrpB: a general biocatalytic platform for synthesis of tryptophan analogues. J. Am. Chem. Soc. 139, 10769-10776 (2017).

22. Maria-Solano, M. A., Iglesias-Fernández, J. \& Osuna, S. Deciphering the allosterically driven conformational ensemble in tryptophan synthase evolution. J. Am. Chem. Soc. 141, 13049-13056 (2019).

23. Zhong, Z., Ravikumar, A. \& Liu, C. C. Tunable expression systems for orthogonal DNA replication. ACS Synth. Biol. 7, 2930-2934 (2018).

24. Lee, M. E., DeLoache, W. C., Cervantes, B. \& Dueber, J. E. A highly characterized yeast toolkit for modular, multipart assembly. ACS Synth. Biol. 4, 975-986 (2015).

25. Watkins, E. J., Almhjell, P. J. \& Arnold, F. H. Direct enzymatic synthesis of a deep-blue fluorescent noncanonical amino acid from azulene and serine. ChemBioChem 21, 80-83 (2020).

26. Boville, C. E., Romney, D. K., Almhjell, P. J., Sieben, M. \& Arnold, F. H. Improved synthesis of 4-cyanotryptophan and other tryptophan analogues in aqueous solvent using variants of Trpb from thermotoga maritima. J. Org. Chem. 83, 7447-7452 (2018).

27. Buller, A. R. et al. Directed evolution mimics allosteric activation by stepwise tuning of the conformational ensemble. J. Am. Chem. Soc. 140, 7256-7266 (2018).

28. Wrenbeck, E. E., Azouz, L. R. \& Whitehead, T. A. Single-mutation fitness landscapes for an enzyme on multiple substrates reveal specificity is globally encoded. Nat. Commun. 8, 1-10 (2017).

29. Dick, M., Sarai, N. S., Martynowycz, M. W., Gonen, T. \& Arnold, F. H. Tailoring tryptophan synthase TrpB for selective quaternary carbon bond formation. J. Am. Chem. Soc. 141, 19817-19822 (2019).

30. Romney, D. K., Sarai, N. S. \& Arnold, F. H. Nitroalkanes as versatile nucleophiles for enzymatic synthesis of noncanonical amino acids. ACS Catal. 9, 8726-8730 (2019).

31. O’Maille, P. E. et al. Quantitative exploration of the catalytic landscape separating divergent plant sesquiterpene synthases. Nat. Chem. Biol. 4, 617-623 (2008).

32. Makałowski, W. \& Boguski, M. S. Evolutionary parameters of the transcribed mammalian genome: An analysis of 2,820 orthologous rodent and human sequences. Proc. Natl Acad. Sci. USA 95, 9407-9412 (1998).
33. Marks, D. S. et al. Protein $3 \mathrm{D}$ structure computed from evolutionary sequence variation. PLoS ONE 6, e28766 (2011).

34. Stiffler, M. A. et al. Protein structure from experimental evolution. Cell Syst. 10, 15-24.e5 (2020)

35. Gibson, D. G. et al. Enzymatic assembly of DNA molecules up to several hundred kilobases. Nat. Methods 6, 343-345 (2009).

36. Gietz, R. D. \& Schiestl, R. H. High-efficiency yeast transformation using the LiAc/SS carrier DNA/PEG method. Nat. Protoc. 2, 31-34 (2007).

37. Ryan, O. W. \& Cate, J. H. D. Multiplex engineering of industrial yeast genomes using CRISPRm. Methods Enzymol. 546, 473-489 (2014). Elsevier Inc.

38. Jung, P. P., Christian, N., Kay, D. P., Skupin, A. \& Linster, C. L. Protocols and programs for high-throughput growth and aging phenotyping in yeast. PLoS ONE 10, e0119807 (2015).

39. Lane, A. N. \& Kirschner, K. The catalytic mechanism of tryptophan synthase from Escherichia Coli. Eur. J. Biochem 129, 571-582 (1983).

40. Goodsell, D. S., Autin, L. \& Olson, A. J. Illustrate: software for biomolecular illustration. Structure 27, 1716-1720.e1 (2019).

41. Eigen, M., McCaskill, J. \& Schuster, P. Molecular quasi-species. J. Phys. Chem 92, 6881-6891 (1988).

\section{Acknowledgements}

This work was funded by NIH NIGMS (1DP2GM119163-01), NSF (MCB 1545158), and the Beckman Young Investigator Award to C.C.L., and the NIH NIGMS (R01GM125887) to F.H.A. The content is solely the responsibility of the authors and does not necessarily represent the official views of funding sources. We thank David Romney, Justin Bois, and other members of the Arnold and Liu groups for thoughtful discussions on experimental design.

\section{Author contributions}

All authors contributed to experimental design and data analysis. G.R. cloned all genetic constructs; set up, performed, and characterized evolution experiments; and carried out yeast growth rate experiments. E.J.W. performed the panel HPLC-MS assay and indole conversion rate measurements on $\operatorname{Tm}$ TrpBs from variant set 2, and P.J.A. analyzed the results. C.E.B. performed in vitro characterizations of $\operatorname{Tm} \operatorname{TrpBs}$ from variant set 1 and performed the thermal shift assay and substrate scope characterizations for $\operatorname{Tm} \operatorname{TrpB}$ variants WT-003-1-A, Q90*-003-1-A, and Tri-100-2-A. P.J.A. performed enzyme kinetics assays for TmTrpB variants WT-003-1-A, Q90*-003-1-A, and Tri-100-2-A. G.R. and C.C.L. wrote the paper with input and contributions from all authors.

\section{Competing interests}

C.E.B and F.H.A. are co-founders of Aralez Bio, focusing on the enzymatic synthesis of unnatural amino acids. All other authors declare no competing interests.

\section{Additional information}

Supplementary information is available for this paper at https://doi.org/10.1038/s41467020-19539-6.

Correspondence and requests for materials should be addressed to C.C.L.

Peer review information Nature Communications thanks the anonymous reviewer(s) for their contribution to the peer review of this work.

Reprints and permission information is available at http://www.nature.com/reprints

Publisher's note Springer Nature remains neutral with regard to jurisdictional claims in published maps and institutional affiliations.

Open Access This article is licensed under a Creative Commons Attribution 4.0 International License, which permits use, sharing, adaptation, distribution and reproduction in any medium or format, as long as you give appropriate credit to the original author(s) and the source, provide a link to the Creative Commons license, and indicate if changes were made. The images or other third party material in this article are included in the article's Creative Commons license, unless indicated otherwise in a credit line to the material. If material is not included in the article's Creative Commons license and your intended use is not permitted by statutory regulation or exceeds the permitted use, you will need to obtain permission directly from the copyright holder. To view a copy of this license, visit http://creativecommons.org/ licenses/by/4.0/.

(C) The Author(s) 2020 